\title{
Speech Identification with Temporal and Spectral Modification in Subjects with Auditory Neuropathy
}

\author{
Vijaya Kumar Name ${ }^{1}$ and C. S. Vanaja ${ }^{2}$ \\ ${ }^{1}$ Department of Audiology, All India Institute of Speech and Hearing, Manasagangothri, Mysore, Karnataka, 570006, India \\ ${ }^{2}$ Department of Audiology \& Speech Language Pathology, Bharati Vidyapeeth University School of Audiology and \\ Speech Language Pathology, Dhankawadi, Pune, Maharashtra 410021, India
}

Correspondence should be addressed to Vijaya Kumar Name, vijaynarne@gmail.com

Received 16 July 2012; Accepted 5 August 2012

Academic Editors: D. C. Alpini, D. Beutner, and M. P. Robb

Copyright ( 2012 V. Kumar Name and C. S. Vanaja. This is an open access article distributed under the Creative Commons Attribution License, which permits unrestricted use, distribution, and reproduction in any medium, provided the original work is properly cited.

Background. The aim of this study was to investigate the individual effects of envelope enhancement and high-pass filtering $(500 \mathrm{~Hz})$ on word identification scores in quiet for individuals with Auditory Neuropathy. Method. Twelve individuals with Auditory Neuropathy (six males and six females) with ages ranging from 12 to 40 years participated in the study. Word identification was assessed using bi-syllabic words in each of three speech processing conditions: unprocessed, envelope-enhanced, and high-pass filtered. All signal processing was carried out using MATLAB-7. Results. Word identification scores showed a mean improvement of $18 \%$ with envelope enhanced versus unprocessed speech. No significant improvement was observed with high-pass filtered versus unprocessed speech. Conclusion. These results suggest that the compression/expansion signal processing strategy enhances speech identification scores - at least for mild and moderately impaired individuals with AN. In contrast, simple high-pass filtering (i.e., eliminating the low-frequency content of the signal) does not improve speech perception in quiet for individuals with Auditory Neuropathy.

\section{Introduction}

Conventional hearing aids amplify acoustic signals to make sounds audible to hearing-impaired individuals. Their basic structure consists of a microphone, an amplifier, a receiver (speaker), and a power supply. The amplifier is the major component that amplifies the input signal. Two types of amplification schemes are typically used in hearing aid design. The first scheme is the linear amplification, in which a set amount of gain is applied to the input signal. In this design, the maximum output is limited by peak clipping, which causes various forms of distortion and reduces the intelligibility and subjective quality of speech. The second scheme is a nonlinear amplification, which reduces gain as the output or input approach maximum values. In this scheme, amplitude compression is implemented by an analog circuit or by a digital processing algorithm to reduce the gain of the instrument when either the input or output exceeds a predetermined level [1]. This type of amplification results in a wider dynamic range, making soft sounds audible without making loud sounds uncomfortably loud [2]. However, amplitude compression also changes the temporal properties of the original speech signal, which may reduce speech intelligibility.

Conventional hearing aids are not effective for all hearing impairments $[3,4]$. The primary function of a conventional hearing aid is to amplify and make the speech signal audible within the hearing threshold and loudness tolerance levels of an individual with hearing loss. These systems are typically effective for hearing impairments caused by a loss of amplification function in the ear, such as with cochlear hearing loss due to outer hair cell loss and/or damage. However, even sophisticated hearing aids are limited in their ability to address other, less common types of hearing loss, such as hearing loss caused by neural fiber removal (via tumor operations), which leave patients with little or no residual hearing, or losses caused by damage to the inner hair cells, neural pathways, or brainstem, which 
affects supra-threshold processing and introduces sound distortion.

Auditory Neuropathy (AN) can involve loss of inner hair cells (IHC), dysfunction of the IHC-nerve synapses, neural demyelination, axonal loss, or possible combinations of the above. Clinically, these pathologies may be mixed with traditional cochlear impairment involving OHC's and/or central processing disorders involving the brainstem and cortex. Because one possible neural mechanism underlying AN is desynchronized discharge of the auditory nerve fibers, AN has also been termed "Auditory Dys-synchrony" [5]. AN can cause sound attenuation, but, more significantly, it causes sound distortion, which cannot be compensated by conventional hearing aids $[3,4]$. Therefore, new signal processing strategies should be developed to specifically address the issue of sound distortion for these participants.

Clinical and psychoacoustic testing on individuals with AN has been conducted to investigate the root causes of sound distortion [6]. Pure-tone audiograms of AN subjects show a global trend opposite to regular hearing impairment-high thresholds at low frequencies but low or relative normal thresholds at high frequencies-implying that amplifying energy at high frequencies or transposing high-frequency components to the low-frequency range may not help. Test results from the temporal modulation transformation function (TMTF) show that individuals with AN have poorer temporal modulation discrimination ability than normal-hearing and other hearing-impaired people. This again implies that conventional hearing aids will not be fully effective for these individuals since their degraded temporal modulation is not being compensated. In addition, data from gap detection tests shows lower gap discrimination ability in individuals with AN than other hearing impairments, which also supports the notion that AN patients have impaired temporal processing abilities. Therefore, it appears that new strategies might be developed based on these clinical and psychoacoustic data to solve the problem of sound distortion in AN.

One strategy directly stemming from the TMTF is to increase the modulation index in each frequency band to compensate for the temporal modulation loss due to desynchronized discharges in the auditory nerve fibers of individuals with AN. This can be implemented over each extracted envelope in each frequency band by directly increasing the amplitude of peaks and decreasing the amplitude of troughs in a local temporal range. However, Fu and Shannon [7] pointed out that enhancement of the envelope using such a "power law expansion scheme" tends to modify consonantto-vowel ratio when the overall RMS of the expanded and control (unexpanded) stimuli are equated and leading to sound distortion in the expanded sound. Apoux et al. [8] applied an envelope enhancement scheme, which enhances the consonantal portion of the signal while attenuating the vowel portion. They observed a significant improvement in identification scores when the envelope-enhanced stimuli were presented to individuals with cochlear hearing loss in the presence of background noise. Research has indicated that "clear speech" also improves speech perception in individuals with cochlear hearing loss. One of factors linked to increased intelligibility of clear speech in cochlear hearing loss is increased consonant-vowel ratio [9-11].

Zeng and Liu [12] reported that subjects with AN showed improved performance in quiet as well as in noise when clear speech was presented. This improvement was attributed to the enhanced temporal envelopes found in clear speech. Starr et al. [13] have shown that (artificially) enhancing the temporal envelope improves consonant identification in quiet for individuals with AN. From the simulation studies it is understood that the reduced ability to follow amplitude variations leads to the loss of distinction between the vowel and consonant causing significant impairment in speech perception $[6,14]$. If this is true, then envelope enhancement should enhance the salient cues for speech perception in individuals with AN, across both quiet and noisy environments. This study was undertaken to investigate this hypothesis in quiet environments.

In addition to the previously identified temporal resolution deficits that typify AN, psychoacoustical studies have demonstrated that individuals with AN have impaired frequency discrimination. It has been reported $[6,15]$ that frequency discrimination is poorer for low frequency sounds and performance improves as the frequency is increased, reaching near normal values at $4000 \mathrm{~Hz}$. Consistent with these findings, Rance et al. [16] and Starr et al. [13] observed that individuals with AN demonstrate better identification for phonemes that lie in relatively high-frequency ranges than for those phonemes that lie in relatively low-frequency ranges. Zeng et al. [17] have hypothesized that eliminating the low-frequency content of speech signal or shifting the low frequency content to high frequencies may improve speech perception and reduce undesirable masking in individuals with AN.

\section{Method}

2.1. Participants. Twelve individuals (six males and six females) who have been diagnosed with AN participated in the study. Table 1 shows the audiological profile of the participants. The age of the participants ranged from 12 to 40 years with a mean of 25 years. All the participants were native speakers of Kannada, a Dravidian language spoken in a southern state of India. The pure-tone average (average of pure tone thresholds at 500,1000 , and $2000 \mathrm{~Hz}, 4000 \mathrm{~Hz}$, and $8000 \mathrm{~Hz}$ ) of the participants ranged from 15 to $55 \mathrm{~dB}$ HL. A majority of the participants had symmetrical hearing loss with rising audiometric configuration. The middle ear acoustic reflexes (both ipsilateral and contralateral) and the auditory brainstem responses were absent in all the participants. None of the participants showed improvement in speech recognition scores with a conventional linear gain behind ear hearing aid.

2.1.1. Ethical Considerations. In the present study, all the testing procedures were approved by institutional review board. The procedures involved in the present study were noninvasive and all the procedures were explained to the patients and their family members before testing, and 
TABLE 1: Audiological profile of individuals with auditory neuropathy.

\begin{tabular}{|c|c|c|c|c|c|}
\hline Subject no. & Age/sex & $\begin{array}{l}\text { Pure-tone average in } \mathrm{dB} \mathrm{HL} \\
\text { (Right ear) }\end{array}$ & $\begin{array}{c}\text { Pure-tone average in } \mathrm{dB} \mathrm{HL} \\
\text { (Left ear) }\end{array}$ & $\begin{array}{l}\text { Speech identification scores } \\
\text { in sound filed }\end{array}$ & $\begin{array}{l}\text { Peripheral } \\
\text { neuropathy }\end{array}$ \\
\hline AN1 & 12 ys/M & 22.50 & 25.5 & 40 & No \\
\hline AN2 & $20 \mathrm{ys} / \mathrm{F}$ & 28.75 & 30.5 & 72 & No \\
\hline AN3 & $15 \mathrm{ys} / \mathrm{F}$ & 26.25 & 25.5 & 20 & Not done \\
\hline AN4 & 39 ys/F & 31.25 & 32.5 & 8 & No \\
\hline AN5 & $12 \mathrm{ys} / \mathrm{M}$ & 40.00 & 42.5 & 40 & No \\
\hline AN6 & $24 \mathrm{ys} / \mathrm{M}$ & 32.50 & 35.5 & 16 & Present \\
\hline AN7 & $27 \mathrm{yr} / \mathrm{F}$ & 26.25 & 29.5 & 68 & No \\
\hline AN8 & $20 \mathrm{yr} / \mathrm{M}$ & 47.50 & 46 & 28 & No \\
\hline AN9 & $18 \mathrm{yrs} / \mathrm{M}$ & 8.75 & 10 & 84 & No \\
\hline AN10 & $20 \mathrm{yrs} / \mathrm{M}$ & 40.00 & 35.5 & 48 & Not done \\
\hline AN11 & $20 \mathrm{yrs} / \mathrm{F}$ & 27.50 & 22.5 & 12 & No \\
\hline AN12 & $19 \mathrm{yrs} / \mathrm{F}$ & 21.25 & 23.5 & 68 & No \\
\hline
\end{tabular}

informed consent has been taken from all the patients and their family members for participating in the study.

2.2. Stimuli. The speech stimuli used in the present study were taken from bi-syllabic wordlists in Kannada, developed by Yathiraj and Vijaylakshami [18]. This test contains four word lists, each with 25 bi-syllabic words, which are phonetically balanced and are equally difficult. The words were spoken in conversational style by a female native speaker of Kannada. They were digitally recorded in an acoustically treated room, on a data acquisition system using $44.1 \mathrm{kHz}$ sampling frequency and 32-bit analog to digital converter.

\subsection{Signal Processing}

(i) Envelope Enhancement. The enhancement procedure used in the present study was similar to that used by Apoux et al. [8] for individuals with cochlear hearing loss. The original speech stimuli, $X(t)$, was expanded/compressed using MATLAB-7.

The signal $X(t)$ was divided into 4 bands using band pass filters (third order Butterworth) with cut-off frequencies of $150-550,550-1550,1550-3550$, and $3550-8000 \mathrm{~Hz}$. The temporal envelopes $E(t)$ were extracted from each band by full-wave rectification and low pass filtering (third-order Butterworth) with a cut-off frequency $32 \mathrm{~Hz}$. This was chosen based on the result of an earlier investigation, where it produced the best outcome among several different cut-off frequencies used [13]. The temporal envelope $E(t)$ was raised to the power of $f$, with $f$ ranging from a highly expansive value $\left(f_{\max }=4\right)$ to a highly compressive value $\left(f_{\min }=0.3\right)$ as a function of the instantaneous envelope amplitude, Ei. The exponent $f$ was computed via a decreasing, exponential function of instantaneous envelope amplitude value $E i$, and was set such that: (i) maximum enhancement $\left(f_{\max }=4\right)$ was applied to the lowest envelope amplitude $E_{\min }$ and (ii) maximum compression $\left(f_{\min }=0.3\right)$ was applied to the highest envelope amplitude value. The formula used for computing the $f$ is given as follow:

$$
f_{i}=e^{-\left(F_{i}-F_{\min }\right) / \tau} \tau\left(f_{\max }-f_{\min }\right)+f_{\min } .
$$

In this equation, $\tau$ was a constant, ( 0.5 for each word) and $E_{\min }$, the minimum envelope amplitude value, was computed over the whole signal duration. A correction factor was then obtained for all the bands by computing the ratio of expanded and original envelope for each sample. The correction factor obtained was then multiplied with the original band pass signal at each corresponding point in time, and finally the resulting bands were added at output and low pass filtered (3rd order Butterworth) with a cutoff frequency of $8000 \mathrm{~Hz}$. The RMS amplitude of the expanded signals was then equated to that of the unprocessed signals. Three word lists were processed for envelope enhancement. Figure 1 shows the waveform of signal with and without envelope enhancement.

(ii) High-Pass Filtering. Bisyllabic words were high-pass filtered (fourth order Butterworth) with a cutoff frequency of $500 \mathrm{~Hz}$. The filtering was done using the MATLAB-7 program. The RMS amplitude of the filtered signals was then equated to that of the unprocessed signals. All four word lists were passed through the filters.

2.4. Procedure. The participants listened to speech tokens individually in a double-walled, acoustically treated room where the ambient levels were within permissible limits [19]. The speech stimuli were played manually from a PC at $44.1 \mathrm{kHz}$ sampling rate and routed to a calibrated [20] diagnostic audiometer (Madsen OB-922 with speaker). The participants listed to the signal from the loudspeaker of the audiometer at a distance of one meter with $0^{\circ}$ azimuth. The presentation level of the stimulus was $40 \mathrm{~dB}$ SL (re: Speech Recognition Threshold). Each participant listened to a total of four lists: one unprocessed, two filtered, and one envelope enhanced. The order of presentation of the 


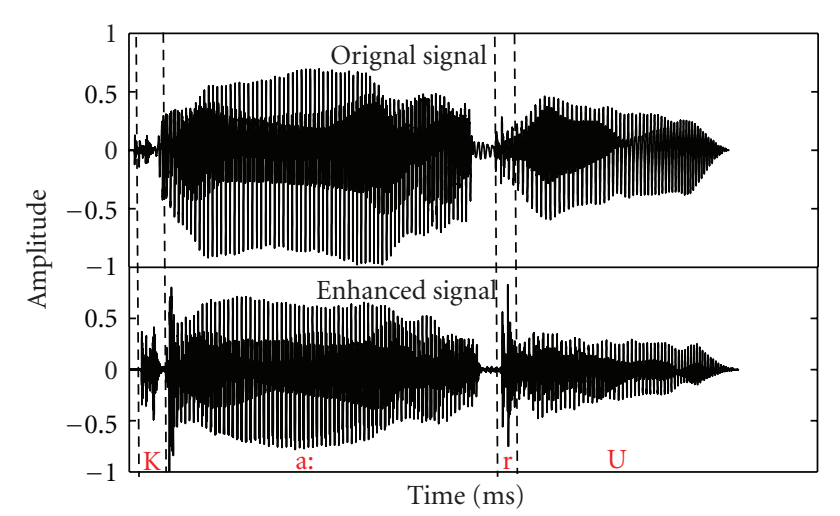

Figure 1: Waveform of the original signal (upper panel) and enhanced signal (lower panel) for word/ka: $\mathrm{r} \mathrm{u} /$. The dotted line indicates the vowel portion of the signal, and striated line indicates the consonantal portion of the signal.

lists was randomized across the participants. Participants had to repeat the speech tokens heard by them. The speech recognition scores were calculated by counting the number of words correctly repeated.

2.5. Data Analysis. Statistical analyses of the data were performed using SPSS-16. Paired sample " $t$ " testing was carried out to assess whether there was a significant difference between the scores obtained in the unprocessed, filtered, and envelope-enhanced conditions. Pearson's product moment correlation coefficient was calculated to investigate if there was a correlation between pure-tone threshold and word identification scores in the three conditions. Because a majority of the participants had symmetrical hearing loss, only right ear thresholds were used for this analysis.

\section{Results}

3.1. Word Identification with Envelope Enhanced Speech. Figure 2 presents the identification scores for unprocessed and envelope-enhanced words for the AN test subjects. The mean identification score for envelope-enhanced speech was $61.3 \%$ with a standard deviation (SD) of $33.2 \%$, whereas the mean identification score for unprocessed speech was $43.6 \%$ with an SD of $27 \%$. The improvement observed for envelope-enhanced speech ranged from $8 \%$ to $36 \%$ with a mean improvement of $18.3 \%$. To assess effect of gender on identification scores in unprocessed and envelope-enhanced condition, a paired sample " $t$ " test was performed and results showed no significant effect of gender on identification for unprocessed $(t=0.44, P=0.66)$ and envelope-enhanced $(t=-0.31, P=0.75)$. As there was a significant effect of gender the data from both males and females is combined for further analysis.

The paired sample " $t$ " test indicates that the improvement observed for envelope enhanced speech was statistically significant $(t=-4.38, P<0.01)$. Pearson's correlation coefficient revealed that there was no significant correlation between word identification scores for the unprocessed signal

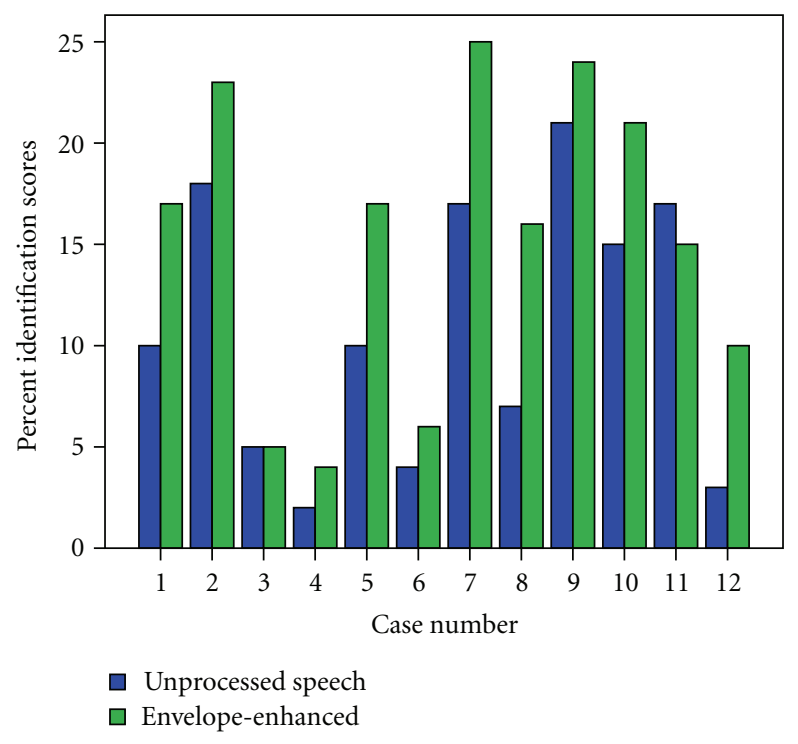

FIGURE 2: Word identification scores for unprocessed (open bar) and envelope-enhanced (filled) speech.

and pure-tone average threshold $(r=-0.37, P=0.6)$. Scores for envelope enhanced speech also did not show a significant correlation with pure-tone average threshold $(r=-0.13$, $P=0.23$ ).

3.2. Word Identification with Filtered Speech. Percent identification scores for all participants are presented in Figure 3. It can be noted from the figure that five participants showed a small improvement in identification with filtered speech, while seven participants showed no improvement or deterioration in performance with filtered speech. The mean identification score for high-pass filtered speech at $500 \mathrm{~Hz}$ was $45.6 \%$ with standard deviation of $25.4 \%$, whereas mean identification score for unprocessed speech was $43 \%$ with standard deviation of $26.3 \%$. To assess effect of gender on identification scores in unprocessed and envelope-enhanced condition, a paired sample " $t$ " test was performed and results showed no significant effect of gender on identification for filtered speech ( $t=0.29, P=0.79)$. As there was significant effect of gender, the data from both males and females is combined for further analysis.

Paired sample " $t$ " testing revealed that the mean difference in scores between unprocessed speech and high pass filtered speech was not statistically significant $(t=1.54, P=$ 0.85 ). Pearson's correlation coefficient revealed that there was no significant correlation between word identification scores for unprocessed speech and pure-tone average threshold $(r=$ $-0.37, P=0.6)$. Scores for envelope enhanced speech also did not show a significant correlation with pure-tone average threshold ( $r=-0.13, P=0.23$ ).

\section{Discussion}

4.1. Word Identification with Envelope-Enhanced Speech. One of the primary goals of the present study was to compare 


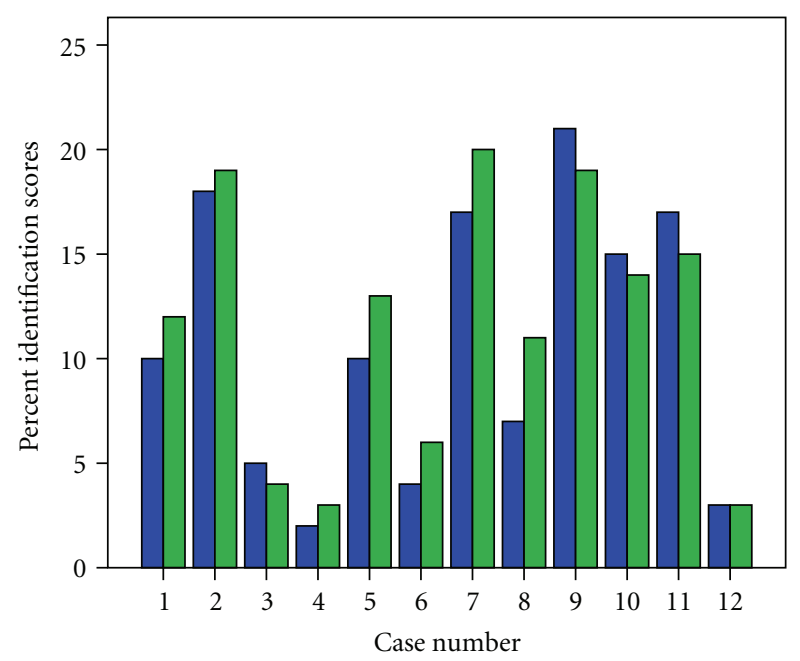

$\square$ Unprocessed speech

$\square$ Filtered speech

FIGURE 3: Word identification scores for unprocessed (open bar) and filtered speech (filled bar).

the word identification scores for unprocessed and envelopeenhanced speech in individuals with AN. The results of the present study reveal that envelope enhanced speech improved word identification in all but four participants with AN. Starr et al. [13] observed similar results for consonant identification in a earlier study. Zeng and Liu [12] have observed similar results for clear speech with AN.

Speech understanding difficulties in individuals with AN are mainly attributed to an impairment in processing the amplitude modulations of the speech signal $[6,15]$. Impaired processing of amplitude variations blurs the distinction between consonant and following vowel, which limits the perception of salient consonantal cues [14]. Dubno and Levitt [21] observed that in both quiet and noise, normalhearing subjects placed more importance on the consonantal energy and consonant-to-vowel ratio, respectively, in identifying the consonants. Studies in normal hearing and those with cochlear hearing loss have shown greater improvement in speech identification in the presence of noise when the processing strategy enhanced the consonantal portion of the signal while attenuating the vowel portion [8]. These observations suggest that when temporal processing is impaired, so too is the ability to identify consonants. By enhancing the amplitude modulations of the speech signal by using the compression/expansion scheme employed in the present study, the consonantal portion of speech signal was also enhanced, leading to an improvement in word identification in individuals with AN.

In the present study four participants did not improve with envelope enhancement. However, these individuals had very poor unprocessed speech identification scores. Zeng et al. [6] and Narne and Vanaja. [15] have demonstrated high levels of correlation between speech identification scores and amplitude modulation detection capabilities. They also reported that some individuals with AN even have difficulty in perceiving amplitude fluctuations as high as $100 \%$. Zeng et al. [6] categorized individuals with AN into different groups based on the TMTF threshold. These groups were mild, moderate, severe, and profound. TMTF thresholds were not assessed in the present study, but it is likely that the degree of TMTF impairment was greater in individuals who showed no or minimal improvement in this study, and that the envelope enhancement was insufficient to overcome this degree of impairment.

Figure 4 depicts the long-term average speech spectrum of unprocessed and envelope-enhanced stimuli, normalized for long-term RMS level. The long-term spectrum was computed using a $50 \mathrm{~ms}$ nonoverlapping Hamming window. The envelope enhanced speech shows 3-5 dB higher energy in the mid-frequency region compared to the unprocessed speech. However, the improved word identification for envelope enhanced speech cannot be attributed to the small increase in intensity in the mid-frequency region, as the correlation analysis between pure-tone thresholds and word identification scores and also the results of hearing aid assessment indicate that audibility was not a factor affecting word identification scores in these individuals. Earlier studies have also reported that audibility is not a factor affecting speech identification scores in a majority of the individuals with AN [21]. In addition, the long-term spectra of clear speech show more energy concentration in the midfrequency region [11]. However, Zeng and Liu [12] attribute the advantage observed for clear speech in individuals with AN to the enhanced envelopes of clear speech. Thus, based on the results of the present study and earlier reports, it can be concluded that envelope enhancement improved word identification in individuals with AN.

4.2. Word Identification with Filtered Speech. The word identification for filtered speech was not significantly different from unprocessed speech. It has been reported in the literature that high pass filtering a speech signal at $500 \mathrm{~Hz}$ does not have major effects on identification scores in normal hearing and those with cochlear hearing loss [22]. Results of the present study clearly demonstrate that simply eliminating the low-frequency information by high pass filtering of the speech signal did not improve the speech perception in individuals with $\mathrm{AN}$ in quiet. Furthermore, filtering of the speech signal caused deterioration in speech identification scores in some individuals with AN. A close look at the data suggests that even individuals with low thresholds at low frequency did not benefit from filtering of the speech signal. An independent sample " $t$ " test showed there is no significant difference $(t=0.39, P=0.73)$ in identification scores for filtered speech between low-frequency hearing loss participants and those with other audiogram patterns. This clearly suggests that simply eliminating the low frequency elements of a signal or emphasizing high frequencies does not compensate for poor speech perception in AN, at least in quiet. This suggests that the cause of their speech understanding difficulties is mainly due to temporal impairment. Further, low frequency hearing loss and poor processing of the signal in low-frequencies is also due to temporal 


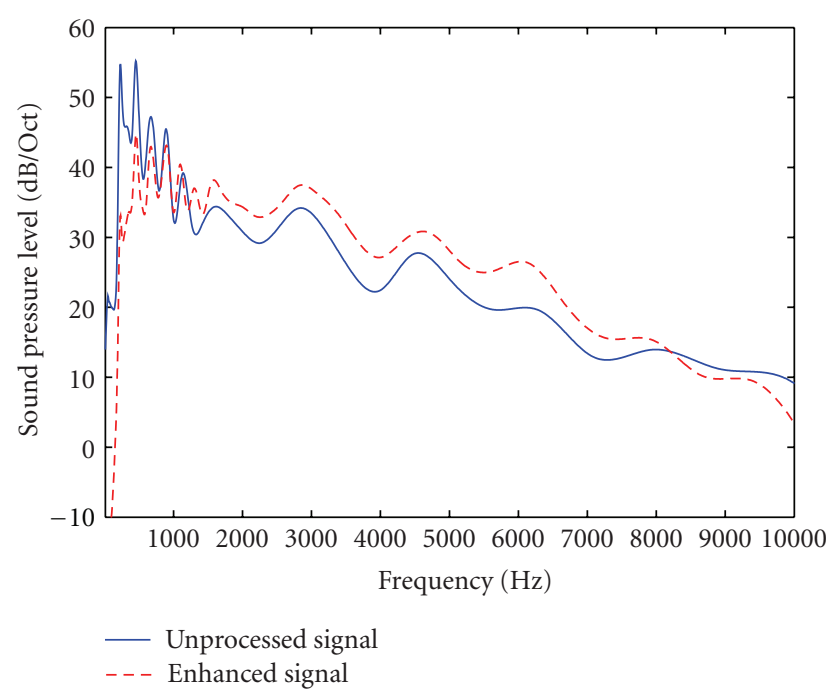

FIGURE 4: Long-term spectrum of enhanced (black) and original (gray) signals for all the stimuli presented.

impairment [23]. However, the importance of eliminating low frequency information in the presence of noise still needs to be investigated.

\section{Conclusion}

Enhancing the speech signal with the compression/expansion scheme, which enhances the consonant portion of the signal, significantly improved speech identification scores in quiet for individuals with $\mathrm{AN}$. In contrast, high pass filtering does not improve the speech identification scores in individuals with AN in quiet. These results suggest that the compression/expansion signal processing strategy enhances speech identification scores-at least for mild and moderately impaired individuals with AN.

\section{Acknowledgments}

The authors extend their sincere thanks to F. Apoux and C. Lorenzi for their technical support in preparing the MATLAB code. They also express their sincere thanks to all participants for their patient cooperation.

\section{References}

[1] W. Davis, "Proportional frequency compression in hearing instruments," Hearing Research, vol. 8, pp. 23-28, 2001.

[2] J. Steinberg and M. Gardner, "Dependence of hearing impairment on sound intensity," Journal of the Acoustical Society of America, vol. 9, pp. 11-23, 1937.

[3] P. Deltenre, A. L. Mansbach, C. Bozet et al., "Auditory neuropathy with preserved cochlear microphonics and secondary loss of otoacoustic emissions," Audiology, vol. 38, no. 4, pp. 187-195, 1999.

[4] G. Rance, B. Cone-Wesson, J. Wunderlich, and R. Dowell, "Speech perception and cortical event related potentials in children with auditory neuropathy," Ear and Hearing, vol. 23, no. 3, pp. 239-253, 2002.

[5] C. I. Berlin, T. Morlet, and L. J. Hood, "Auditory neuropathy/dyssynchrony its diagnosis and management," Pediatric Clinics of North America, vol. 50, no. 2, pp. 331-340, 2003.

[6] F. G. Zeng, S. Oba, S. Garde, Y. Sininger, and A. Starr, "Temporal and speech processing deficits in auditory neuropathy," NeuroReport, vol. 10, no. 16, pp. 3429-3435, 1999.

[7] Q. J. Fu and R. V. Shannon, "Effects of amplitude nonlinearity on phoneme recognition by cochlear implant users and normal-hearing listeners," Journal of the Acoustical Society of America, vol. 104, no. 5, pp. 2570-2577, 1998.

[8] F. Apoux, N. Tribut, X. Debruille, and C. Lorenzi, "Identification of envelope-expanded sentences in normal-hearing and hearing-impaired listeners," Hearing Research, vol. 189, no. 12, pp. 13-24, 2004.

[9] M. A. Picheny, N. I. Durlach, and L. D. Braida, "Speaking clearly for the hard of hearing. I. Intelligibility differences between clear and conversational speech," Journal of Speech and Hearing Research, vol. 28, no. 1, pp. 96-103, 1985.

[10] M. A. Picheny, N. I. Durlach, and L. D. Braida, "Speaking clearly for the hard of hearing. II: acoustic characteristics of clear and conversational speech," Journal of Speech and Hearing Research, vol. 29, no. 4, pp. 434-446, 1986.

[11] J. C. Krause and L. D. Braida, "Acoustic properties of naturally produced clear speech at normal speaking rates," Journal of the Acoustical Society of America, vol. 115, no. 1, pp. 362-378, 2004.

[12] F. G. Zeng and S. Liu, "Speech perception in individuals with auditory neuropathy," Journal of Speech, Language, and Hearing Research, vol. 49, no. 2, pp. 367-380, 2006.

[13] A. Starr, D. McPherson, J. Patterson et al., "Absence of both auditory evoked potentials and auditory percepts dependent on timing cues," Brain, vol. 114, no. 3, pp. 1157-1180, 1991.

[14] R. Drullman, J. M. Festen, and R. Plomp, "Effect of temporal envelope smearing on speech reception," Journal of the Acoustical Society of America, vol. 95, no. 2, pp. 1053-1064, 1994.

[15] V. K. Narne and C. S. Vanaja, "Effect of envelope enhancement on speech perception in individuals with auditory neuropathy," Ear and Hearing, vol. 29, no. 1, pp. 45-53, 2008.

[16] G. Rance, C. McKay, and D. Grayden, "Perceptual characterization of children with auditory neuropathy," Ear and Hearing, vol. 25, no. 1, pp. 34-46, 2004.

[17] F. G. Zeng, Y. Y. Kong, H. J. Michalewski, and A. Starr, "Perceptual consequences of disrupted auditory nerve activity," Journal of Neurophysiology, vol. 93, no. 6, pp. 3050-3063, 2005.

[18] A. Yathiraj and C. S. Vijayalakshmi, Auditory Memory Test. A Test Developed at the Department of Audiology, All India Institute of Speech and Hearing, Mysore, India, 2005.

[19] American National Standards Institute, "Specification for audiometers,” Tech. Rep. ANSI S3.6-1996, American National Standards Institute, New York, NY, USA, 1996.

[20] American National Standards Institute, "Maximum permissible ambient noise for audiometric rooms," Tech. Rep. ANSI S3.1-1999, American National Standards Institute, New York, NY, USA, 1999.

[21] J. R. Dubno and H. Levitt, "Predicting consonant confusions from acoustic analysis," Journal of the Acoustical Society of America, vol. 69, no. 1, pp. 249-261, 1981.

[22] B. W. Y. Hornsby and T. A. Ricketts, "The effects of hearing loss on the contribution of high- and low-frequency speech information to speech understanding. II. Sloping hearing 
loss," Journal of the Acoustical Society of America, vol. 119, no. 3, pp. 1752-1763, 2006.

[23] Y. Sininger and S. Oba, "Patients with AN: who are they and what can they hear?" in AN: A New Perspective on Hearing Disorders, Y. Sininger and A. Starr, Eds., pp. 15-35, SingularThomson Learning, San Diego, Calif, USA, 2001. 


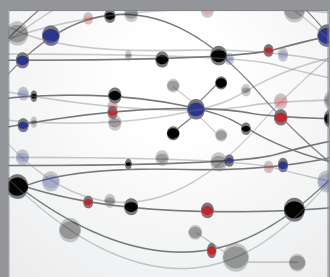

The Scientific World Journal
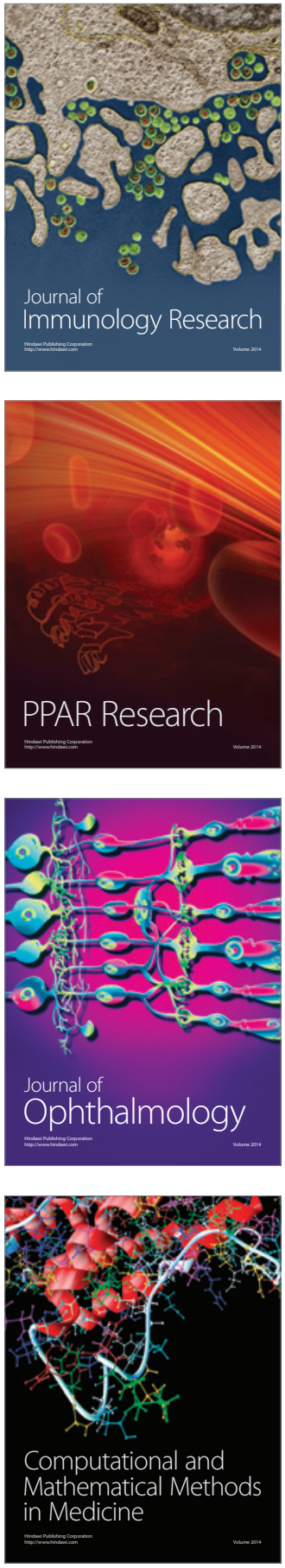

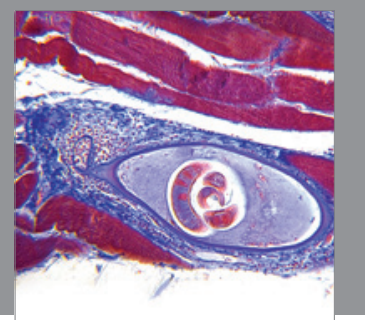

Gastroenterology

Research and Practice
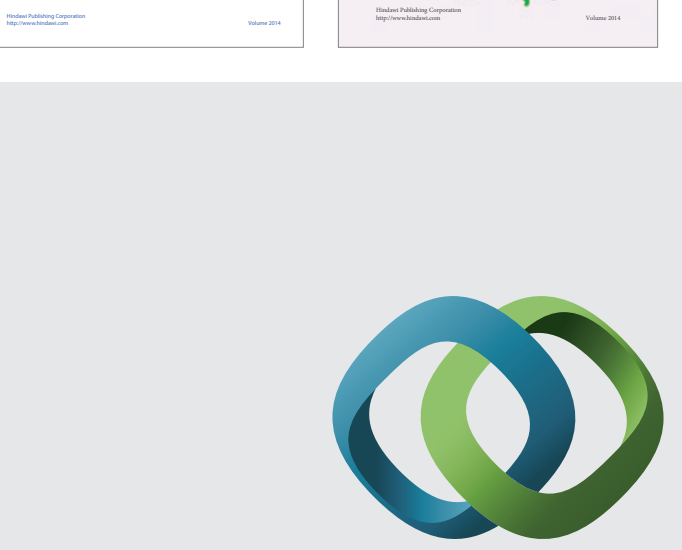

\section{Hindawi}

Submit your manuscripts at

http://www.hindawi.com
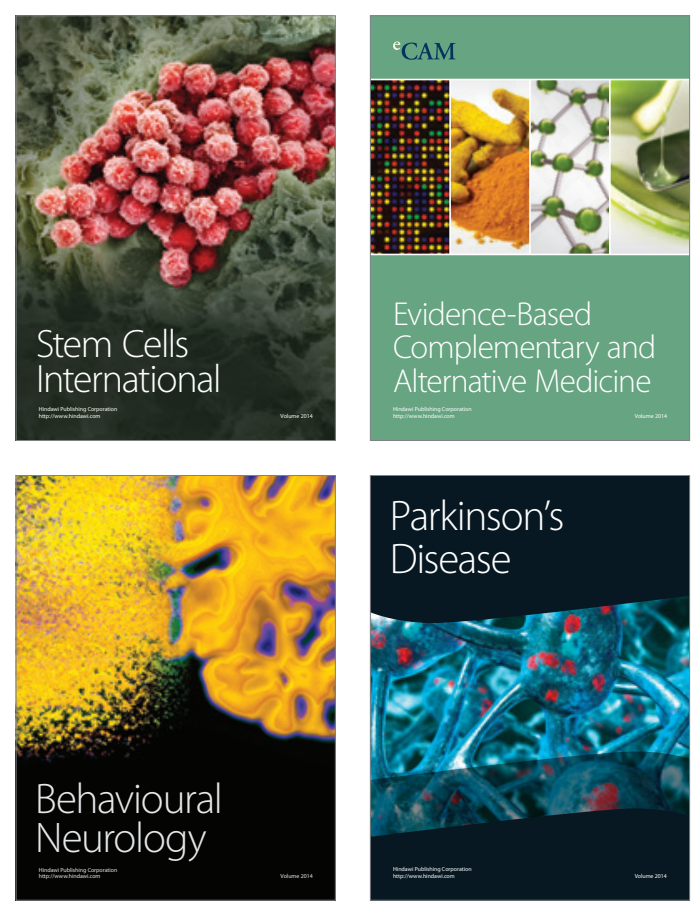

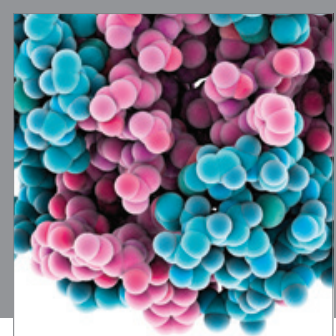

Journal of
Diabetes Research

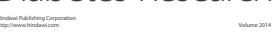

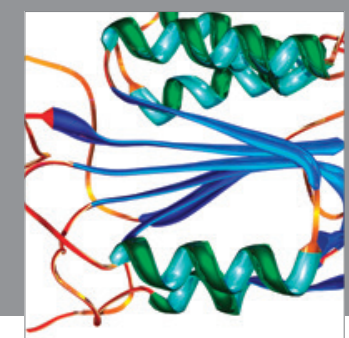

Disease Markers
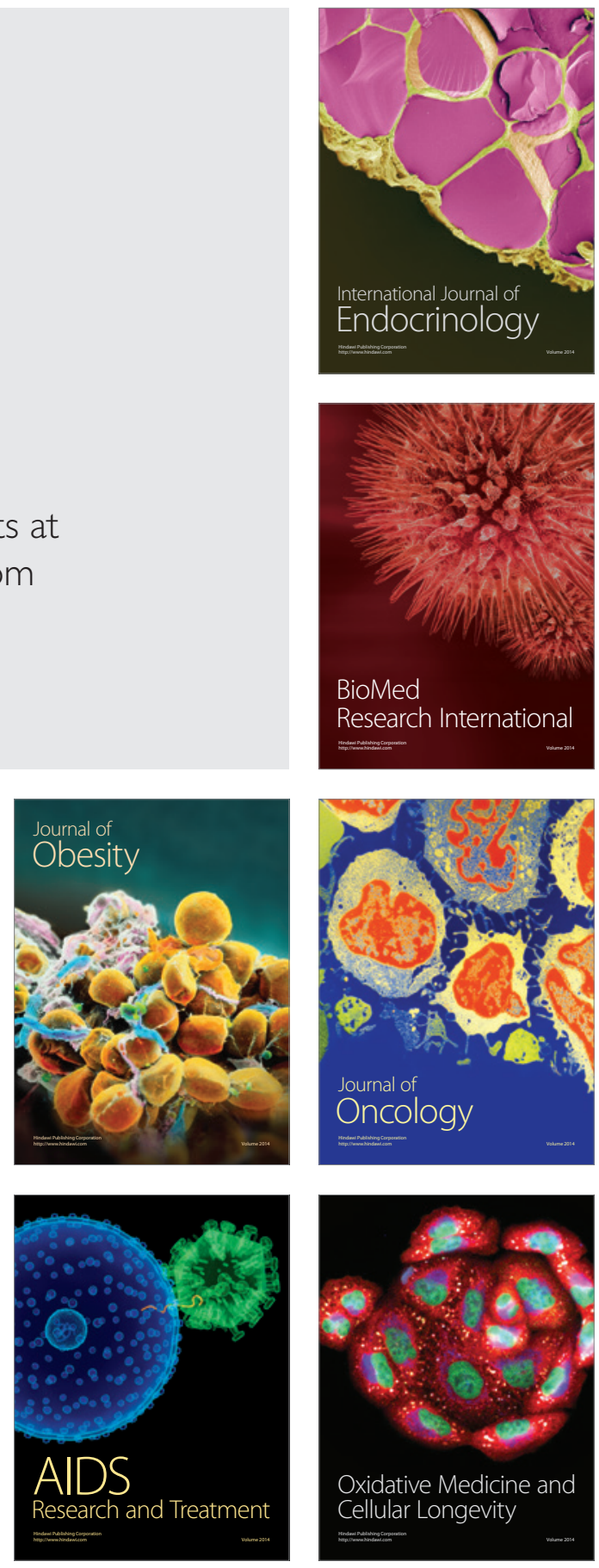